\title{
HOW CHARLES EHRESMANN'S VISION OF GEOMETRY DEVELOPED WITH TIME
}

\author{
ANDRÉE C. EHRESMANN \\ Faculté de Mathématique et Informatique, Université de Picardie Jules Verne \\ 33 rue Saint-Leu, 80039 Amiens, France \\ E-mail: ehres@u-picardie.fr
}

\begin{abstract}
In the mid fifties, Charles Ehresmann defined Geometry as "the theory of more or less rich structures, in which algebraic and topological structures are generally intertwined". In 1973 he defined it as the theory of differentiable categories, their actions and their prolongations. Here we explain how he progressively formed this conception, from homogeneous spaces to locally homogeneous spaces, to fibre bundles and foliations, to a general notion of local structures, and to a new foundation of differential geometry based on groupoids of jets and their actions. These successive generalizations led him to a turning point in the late fifties toward a categorical framework to which he devoted the rest of his life.
\end{abstract}

First of all, I would like to thank the organizers of this Conference for having dedicated it to the memory of Charles. During all his life, my husband thought about Geometry as the foundation of Mathematics. However his idea of Geometry evolved with time, and in the second part of his work, from the late fifties on, it came to include very abstract notions in category theory. Initially they were suggested by geometrical situations but later developed in a general setting, as he hoped they would allow for the unification of several domains into a simple and harmonious framework. As he said in an address given in Lawrence in 1966 [O III, p. 759]*:

"Mathematics is very akin to Art; a mathematical theory not only must be rigorous, but it must also satisfy our mind in quest of simplicity, of harmony, of beauty... For the Platonists among the mathematicians, the motivation of their work lies in this search for the true structure in a given situation and in the study of such an abstract structure for itself... Mathematics is a never finished creation, which has not to justify its existence by the importance and the expanding number of its applications... It is the key for the understanding of the whole Universe".

*All references are given to "Charles Ehresmann: Oeuvres complètes et commentées" (Amiens, 1980-83), denoted by $\mathrm{O}$, in the 4 parts (7 volumes) of which are reprinted all his papers, with updating comments. 
This citation reveals Charles' vision of Mathematics and explains why all his life has been devoted to them. He knew how to communicate his enthusiasm in long discussions and (not very formal) lectures in which he always tried to convey his ideas by drawing figures, for he thought that geometrical insights are most illuminating. So it might be of some interest to point out how his vision of Geometry progressively changed.

What is Geometry for Charles? In the "Notice" Charles wrote for his candidature to the Paris University in 1955 [O I, p. 471], he describes the genesis of his research and its development up to this date, which gives some clues to the turning-point from Geometry toward category theory he began around this time. At the end, he proposes a "large" definition of Geometry as "the theory of more or less rich structures, in which are generally intertwined algebraic and topological structures", obtained by generalization of elementary geometry seen as the study of a topological space $\left(\mathbb{R}^{3}\right)$ equipped with the action of a group of transformations. First the group is replaced by a pseudogroup of transformations which act only on some sub-spaces of the topological space; these sub-spaces form the open sets of a topological structure, so that the pseudogroup has an underlying topological structure. Whence the notion of a local structure, which is still generalized, and enriched by infinitesimal notions. It is these successive generalizations, which abut to the important notions of category theory to which Charles devoted his life from 1957 to his death in 1979 that give the thread of Charles' works.

I have no time here to recall the main results of Charles, even if I restricted myself to his papers on Geometry taken in its usual meaning; anyway they are well-known and still easily accessible, for instance in the 7 volumes of his complete works [O]. My aim is only to indicate the development of his ideas up to his "conversion" to category theory (that was not always well understood by his colleagues!).

Homogeneous and locally homogeneous spaces. Charles became interested in Geometry under its different aspects during his years at the "Ecole Normale Supérieure", when he read the works of Sophus Lie on the advice of Ernest Vessiot, and followed Elie Cartan's lectures on Riemannian spaces which gave him a glimpse on new directions in differential geometry.

His Thesis, obtained in 1934 [O I, p. 3] was supervised by Elie Cartan for whom he kept a great admiration during all his life. Written during a two years stay in Princeton, it is devoted to the topology of some homogeneous spaces, and it still remains a reference on Grassmann manifolds. Their homology and Poincaré groups are determined thanks to a powerful and then original method using cellular decompositions, similar to those considered later on in the theory of CW-complexes.

The same method is also applied to more general manifolds in a paper following his thesis [O 1, P. 55] which in fact formed the second part of his thesis.

His following important paper [O I, p. 87] is devoted to Lie locally homogeneous spaces. He was prompted to consider this notion by the locally Euclidean spaces studied by Elie Cartan in "Leçons sur la géométrie des espaces de Riemann" and by ideas presented in Veblen and Whitehead's book "The Foundations of Differential Geometry". 
The Klein program defined a geometry by the data of a group operating on a space. To define a locally homogeneous space, the group is replaced by a local continuous group (or germ of group), and the space is obtained by gluing together more elementary spaces on which this local group acts, the gluing respecting the structure. Charles proved that such a Lie locally homogeneous space which is compact and locally connected is equivalent to a Lie homogeneous space. In particular, he studies locally projective spaces and locally affine spaces. This paper is important by its later developments, for it conducted Charles to a general definition of a pseudogroup of transformations and of the associated "local structures", the avenue through which he later came to category theory, as we'll explain later on.

Fibre bundles and foliations. In the early forties, Charles introduced the notion of a fibre bundle independently from Steenrod, while the war had broken communications between France and the United States. He develops a general theory in a series of Notes from 1941 to 1944 [O I, p. 310-321], defining locally trivial principal bundles and their associated fibre bundles.

Among the main results, let us note Lifting of Homotopy Theorems and the exact sequence associated to a fibration [O I, p. 105], generalizing theorems proved in special cases in his former papers.

The problem of restricting the structural group is raised and solved in several instances, for instance, for the tangent bundle on a differentiable manifold [O I, p. 133]; as a by-product, he proved that, if the Universe of Relativity is compact, its Euler-Poincaré characteristic must be zero (he was proud that this result [O I, p. 319] brought him his first invitation to Rio de Janeiro in 1952). Other examples he considers are the almost complex, quaternionian or hermitian manifolds, studied in the theses of P. Libermann and $\mathrm{Wu}$ Wen Tsun.

To study manifolds equipped with a completely integrable field of contact elements [O I, p. 322], Charles introduced the theory of a foliated manifold [O I, p. 155], which was developed by Reeb whose thesis is a reference in this domain. Later on, Charles defined more general foliations and adapted the notion of holonomy and the stability theorems to locally simple foliations [O I, p. 370]. These results are refined in a substantial paper written in 1961 [O II, p. 563], unfortunately ignored by most specialists.

Pseudogroups of transformations and local structures. Lie locally homogeneous spaces, topological manifolds, differentiable or analytic manifolds, but also fibre bundles and foliated manifolds, are structures constructed by gluing together more elementary structures of the same specific type in a well-defined sense. In his quest for simplicity and harmony, Charles has searched for a unifying theory.

At this end, making more precise ideas of Veblen-Whitehead, he gives the definition of a pseudogroup of transformations and of the associated structures. This notion is first defined in his 1947 paper on fibre bundles [O I, p. 133], and is generalized in following papers. For instance a fibre bundle becomes a structure associated to an appropriate pseudogroup of transformations on the product of its basis by the fibre. 
Roughly a pseudogroup of transformations $G$ on a set $E$ is formed by a set of 1-1 mappings between sub-sets of $E$ closed under inversion, composition, and such that $g$ belongs to $G$ if it glues elements of $G$. Then the domains of the transformations in $G$ generate a topology on E. A structure associated to $G$ on a set $V$ is obtained by gluing together the images of charts of a complete atlas compatible with $G$ (meaning that the change of charts pertains to $G$ ).

In particular given a group of automorphisms of a topological space $E$, their restrictions to the open sub-spaces form a pseudogroup of transformations on $E$, and the associated structures are the structures locally equivalent to $E$ (equipped with the structure defined by $G$ ).

In 1952, Charles proposes a more general theory of "local structures" in the frame of Bourbaki's species of structures [O I, 352, 411]. The local automorphisms of such a local structure $S$ form a pseudogroup of transformations. Conversely any pseudogroup of transformations $G$ gives rise to local structures, namely the structures associated to it, which are defined by complete atlases having their changes of charts in $G$.

Foundations of Differential Geometry. Bundle theory has important applications in Differential Geometry. In particular, the differentiable bundles are an appropriate setting for the study of infinitesimal connections, as it is shown in [O I, p. 179, 233].

But Charles was unsatisfied with notations on differentials, and this prompted him to introduce a coordinate-free representation, namely the infinitesimal jets. He defined bundles of jets, in the holonomic, semi-holonomic and non-holonomic cases [O I, p. 343369] which led him to give a modern foundation to Differential Geometry (now folklore). In particular he developed a beautiful theory of prolongations of differentiable manifolds in the 3 cases, holonomic and semi-holonomic jets (not yet fully exploited) simplify several problems involving differential systems (to be compared with sprays).

Infinitesimal structures (which generalize geometrical objects) and their covariants, $G$-structures and their associated pseudogroups are introduced, and he raises the local equivalence problem for $G$-structures, studied in the thesis of P. Libermann.

Let us note that the coordinate-free handling of differential geometry initiated by Charles has been fundamental in the more recent development of Synthetic Differential Geometry in the categorical frame of topos theory. For instance Kock recognizes it has strongly influenced him.

The first step toward categories: the groupoids. In his notes on Differential Geometry of the early fifties, Charles makes an extensive use of the notion of a groupoid (in the sense introduced by Brandt in 1926); as such a groupoid can be defined as a category in which every morphism is invertible, it is his first step toward category theory (though he did not realize it at this time).

In fact he had already used the term groupoid in his 1950 paper on connections [O I, p. 179], where he says that the isomorphisms from fiber to fibre of a fibre bundle form a groupoid $\mathrm{HH}^{-1}$. In 1952 [O I, 355], he defines the actions of this principal groupoid on the bundle and uses it, for instance to define covariant maps. 
Groupoids of infinitesimal jets, with their differentiable structure, as well as their operations on manifolds, become an essential tool for the study of prolongations of manifolds and of fibre bundles. In his view at this time, Differential Geometry consists in the study of the spaces on which groupoids of jets act and of their prolongations.

Though he also describes the general composition law on jets and indicates its properties, that amounts to define the category of jets, he does not use the term (he told me that the link with categories was later pointed to him by Constantino de Barros).

In fact, the turning point came in the late fifties, when Charles wrote 2 important papers which initiate his work in category theory and from which many of his papers of the following ten years are more or less directly issued. They provide a unifying setting in the category framework to improve and generalize his former results in several ways.

Species of local structures. In 1951 [O 1, p. 153], Charles had already mentioned that a pseudogroup of transformations is a particular groupoid, and that only the open sets of the associated topology are used, not the points, whence the idea of replacing the pseudogroup of transformations by a groupoid and the topology by a paratopology (i.e., a complete distributive lattice). Let us remark that "topologies without points" have later gained a great importance, since paratopologies are extensively used, under the name of locales, in connection with Topos theory.

This idea is formalized in the 1957 seminal paper "Gattungen von lokalen Strukturen" [O II, p. 126], the first one written in a categorical framework.

The pseudogroup of transformations is replaced by a local groupoid and even, more generally, by a local category, that is a category equipped with a "local" order compatible with its structure (in modern term it is a category internal to a category of locales).

He describes how such a local category can act on a space, determining a species of local structures on it. This species is complete if it satisfies a gluing property (sheaf axiom).

The main result of the paper is the Complete Enlargement Theorem (or Associated Sheaf Theorem) which constructs the complete species of local structures associated to a given species, by a process generalizing the gluing process used to construct the local structures associated to a pseudogroup of transformations. Various applications are given.

Locally trivial groupoids and associated bundles. The following paper in 1959 [O I, p. 237] transposes the theory of fibre bundles in the categorical setting.

In former papers [O I, 355, 367], Charles had noted that the groupoid $H^{-1}$ of isomorphisms between fibres of a fibre bundle has a differentiable structure compatible with its composition, and that it acts on the associated bundles. And he had defined its prolongations, used for example to define higher order connection elements [O I, p. 233]. Now he characterizes the properties of such a principal groupoid.

First he defines the notions of a topological or differentiable category and of its actions, which he proposes to consider as defining generalized fibre spaces.

To recover the principal groupoids, a topological groupoid must be locally trivial; it means that each unit $x$ has an open neighbourhood on which there exists a local section $s$ of the codomain map such that dom $o s$ is the constant map on $x$. 
Charles proves that the theory of locally trivial groupoids is equivalent to that of principal bundles, and that the spaces on which such a groupoid $K$ acts correspond to the associated fibre bundles.

In a 1959 paper [O I, p. 421], he generalizes to differentiable groupoids the theorem he had proved in a Note in 1958 [O I, p. 374], namely that the largest group of transformations included in a Lie pseudogroup of finite type is a Lie group.

Conclusion. In the sixties, Charles wrote only 4 brief papers on Differential Geometry, all in the categorical frame, in which he developed a theory of prolongations of differentiable categories and of their actions (generalizing that of manifolds and bundles). His last views on Differential Geometry are summarized in the abstract [O I, p. 271] of the lecture he gave during a Conference on categories we organized in Amiens in 1973: it is the study of differentiable categories, of their actions on manifolds and of their prolongations.

The topological and differentiable categories led Charles in 1963 to the general notion of internal categories in a category, and of internal actions, and in 1968 to the theory of sketched structures and of completions of categories and functors. In all these more abstract works (reprinted in Parts II and IV of his "Oeuvres"), the initial motivation was often of a geometric nature, even if it is not always apparent in the paper.

Thus the geometric part of Charles' work has been fundamental, not only for the important results he obtained in it, particularly the study of fibre bundles and foliated spaces and the foundations of differential geometry on jets, but also because it gave a support for the more general notions he developed in category theory and for their many applications both in Physics, in Computer Science, and, more recently, in Systemics and Biology. To cover all these aspects, the Będlewo geometrically centred conference was followed in October by the international conference "Charles Ehresmann : 100 ans" organized in Amiens, which concentrated more particularly on Category Theory and multidisciplinary applications. 